\title{
Avaliação e análise de trilha do valor do leite nos estados do Paraná e Rio Grande do Sul
}

\section{Evaluation and path analysis the value of milk in stats of Paraná and Rio Grande do Sul}

\author{
Cleiton Luiz Tonello ${ }^{1 *}$; Leonir Bueno Ribeiro ${ }^{1}$; Orlando Rus Barbosa ${ }^{2}$; \\ Carlos Eduardo Furtado ${ }^{2}$; Carla Franciele Höring ${ }^{3}$; Maicon Carard ${ }^{4}$
}

\begin{abstract}
Resumo
O objetivo deste trabalho foi avaliar o comportamento e análise de trilha da série histórica do valor do leite nos estados do Paraná e Rio Grande do Sul. O banco de dados foi composto das séries históricas dos valores pagos ao litro de leite em reais, índice geral de preços, taxa de câmbio, valor do litro de leite em dólar, valor da saca de milho, soja e trigo em reais para os estados do Paraná e Rio Grande do Sul. A análise estatística dos dados envolveu análise de trilha e análise de variância das médias, pelo teste de Tukey ao nível de 5\% de probabilidade. Observou-se que o maior valor histórico do valor do leite no estado do Rio Grande do Sul foi para os anos de 2007 com média de R $\$ 0,60$ e 2008 com valor médio de $\mathrm{R} \$ 0,64$. O valor do litro de leite no estado do Paraná foi significativamente maior $(\mathrm{P}<0,05)$ em 2007 e 2008 com médias de R \$ 0,61 e R \$ 0,64. Foram observadas diferenças significativas nos valores médios mensais do valor pago ao litro de leite nos estados. O valor da saca de trigo no estado do Paraná teve um elevado efeito direto de correlação de 0,7698 no valor do leite e o valor da saca de soja apresentou um efeito direto negativo no valor do leite. Observou-se variação da remuneração ao litro de leite produzido nos estados do Paraná e Rio Grande do Sul e que os efeitos diretos e indiretos das variáveis analisadas tiveram correlação com a variável Rleite.
\end{abstract}

Palavras-chave: Remuneração, efeitos, soja

\begin{abstract}
The objective of this study was to evaluate the conduct and path analysis the series of historical value of milk in stats of Parana and Rio Grande do Sul. The database was composed of historical series of amounts paid to the litre of milk in real, general price index, exchange rate, value of a litre of milk in dollars, value of the bag of maize, soya and wheat in real draws for the states of Parana and Rio Grande do Sul. Statistical analysis of the data involved path analysis and analysis of variance average, the test Tukey at 5\% probability. It was observed that the greatest historical value of the value of milk in the state of Rio Grande do Sul was for the years 2007 with an average of R\$ 0.60 and 2008 with average value of $R \$ 0.64$. The value of a litre of milk in the state of Parana was significantly higher $(\mathrm{P}<0.05)$ in 2007 and 2008 with averages of $R \$ 0.61$ and $R \$ 0.64$. There were significant differences in monthly average values of the amount paid to the litre of milk in the states. The value of the bag of wheat in the state of Parana had a high of 0.7698 direct effect correlation on the value of milk and the value of a bag of soybeans had direct negative effect on the value of milk. There was a variation of the remuneration litre of milk produced in the states of Parana and Rio Grande do Sul and that the direct and indirect effects of variables were correlated with the variable Rmilk.
\end{abstract}

Key words: Remuneration, effects, soya

Programa de pós-graduação em Zootecnia da Universidade Estadual de Maringá - UEM. E-mail: cleitontonello@msn.com

Departamento de Zootecnia, Universidade Estadual de Maringá - UEM.

Programa de pós-graduação em Agronomia da Universidade Estadual de Londrina - UEL.

Zootecnista pela Universidade Estadual do Oeste do Paraná - UNIOESTE.

Autor para correspondência 


\section{Introdução}

A produção de leite está distribuída por todo o país e a heterogeneidade do processo produtivo é marcante. Os produtores especializados investem em tecnologia, usufruem das economias de escala e diferenciam seu produto, recebendo mais pelo volume produzido e pela qualidade alcançada. $\mathrm{O}$ agronegócio do leite ocupa posição de destaque na economia brasileira, sendo grandes as expectativas, nesta década, de continuarmos o crescimento da produção e da produtividade, com índices maiores do que aqueles que têm sido alcançados em anos recentes (VILELA, 2002).

O Brasil é o quinto maior produtor de leite do mundo e cresce a uma taxa anual de $4 \%$, superior à de todos os paises que ocupam os primeiros lugares. No ano passado, o Valor Bruto da Produção Agropecuária, foi de 36 bilhões de dólares. Destes, aproximadamente 15 bilhões de dólares são de produtos pecuários, tendo o leite posição de destaque, com o valor de 2,8 bilhões, ou $18 \%$ do Valor Bruto da Produção Pecuária, superado apenas pelo Valor da Produção da carne bovina (VILELA, 2002; GOMES, 2001). O custo de produção do leite é muito influenciado pelo preço do milho e soja, pois tanto o milho quanto a soja são componentes básicos na fabricação de concentrados da ração fornecida aos animais.

As correlações não são medidas de causa e efeito e a interpretação direta das suas magnitudes pode resultar em equívocos na estratégia de seleção, pois correlação alta entre dois caracteres pode ser resultados do efeito, sobre estes, de um terceiro caráter ou de um grupo de caracteres (DUNTEMAN, 1984), sendo pois necessário o emprego de metodologias específicas, como as correlações parciais, correlações canônicas e análise de trilha (FALCONER, 1987; CRUZ; REGAZZI, 1997).

A análise de trilha, desenvolvida por Wright (1921, 1923) consiste no estudo dos efeitos diretos e indiretos de caracteres independentes explicativos sobre uma variável dependente principal básica, cujas estimativas são obtidas por meio de equações de regressão, em que as variáveis são previamente padronizadas. O objetivo deste trabalho foi realizar uma avaliação histórica e análise de trilha do valor do leite nos estados do Paraná e Rio Grande do Sul.

\section{Material e métodos}

O experimento foi realizado no Departamento de Zootecnia da Universidade Estadual de Maringá - UEM, situada na cidade de Maringá, Paraná. O banco de dados foi composto das séries históricas dos valores pagos ao litro de leite para o produtor em reais (Rleite), índice geral de preços - disponibilidade interna (IGP-DI), taxa de câmbio (Câmbio), valor do litro de leite em dólar (Usleite), valor da saca de milho em reais (Rmilho), valor da saca de soja em reais (Rsoja) e valor da saca de trigo em reais (Rtrigo) para os estados do Paraná e Rio Grande do Sul.

Os dados foram obtidos por meio de consulta a base de dados do Cepea (Centro de Estudos Avançados em Economia Aplicada), Ipea (Instituto de Pesquisa Econômica Aplicada), Deral (Departamento de Economia Rural), Cooperativas Agroindustriais e Associações leiteiras do Paraná e Rio Grande do Sul. O período de análise para os estados do Rio Grande do Sul e Paraná foram de Janeiro de 1999 à em Dezembro de 2008.

A análise estatística dos resultados foi feita pela análise de trilha através do estudo do desdobramento do coeficiente de correlação entre variável dependente, valor do leite em reais e variáveis independentes (índice geral de preços Disponibilidade Interna, taxa de câmbio, valor da soja em reais, valor do milho em reais e valor do trigo em reais) em efeitos diretos e indiretos. Os resultados da análise de trilha foram interpretados conforme indicado por Vencovsky e Barriga (1992), em que, basicamente, i) coeficientes de correlação e efeitos diretos (coeficientes de trilha) altos indicam que estas variáveis independentes 
explicam grande parte da variação da variável básica e que ii) coeficientes de correlação positivos (ou negativos), mas o efeito direto é de sinal diferente ou neglingenciável, variáveis com maiores efeitos indiretos devem ser consideradas simultaneamente para explicarem a variação da variável básica.

A análise estatística dos dados envolveu a análise de trilha e a análise de variância das médias, pelo teste de Tukey ao nível de 5\% de probabilidade, utilizando-se o programa computacional SAEG 9.1 (UNIVERSIDADE FEDERAL DE VIÇOSA, 2007).

\section{Resultados e discussão}

A primeira análise realizada é a verificação do comportamento visual da série de preços do leite recebidos pelos produtores, estado do Rio Grande do Sul e Paraná. Pela Figura 1, nota-se que a série de preços do leite no estado do Rio Grande do Sul possui um comportamento irregular ascendente até atingir seu nível máximo no mês de agosto de 2007, em que o preço do leite atingiu $\mathrm{R} \$ 0,78$. A partir dessa data, a série teve um movimento de queda irregular e uma pequena recuperação no mês de março de 2008, logo após, reiniciou nova queda no valor recebido pelos produtores. Analisando a distribuição histórica da série de preços do leite, pode-se inferir visualmente que a série não é normalmente distribuída.

\section{Paraná}

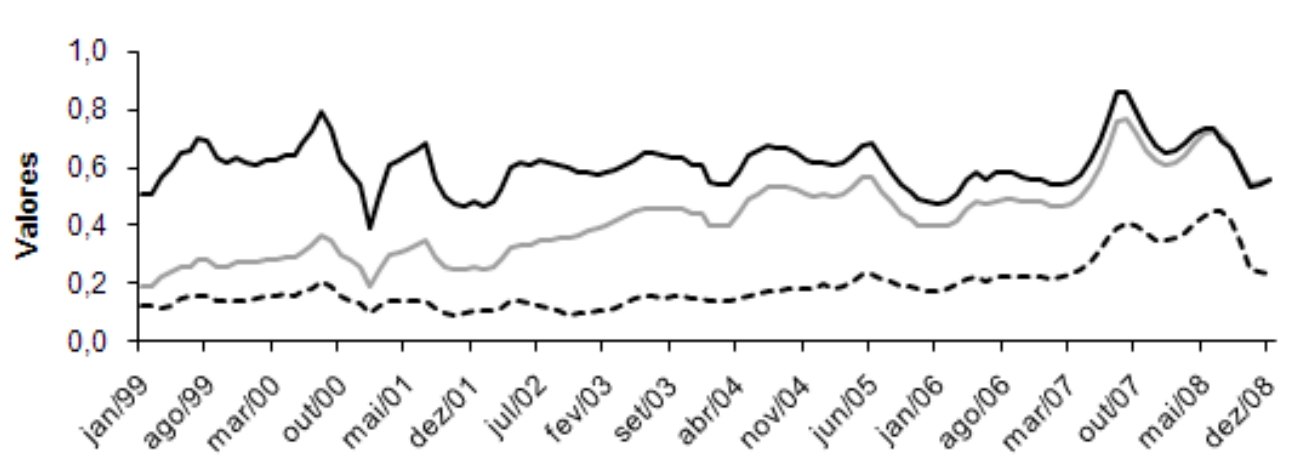

Rio Grande do Sul

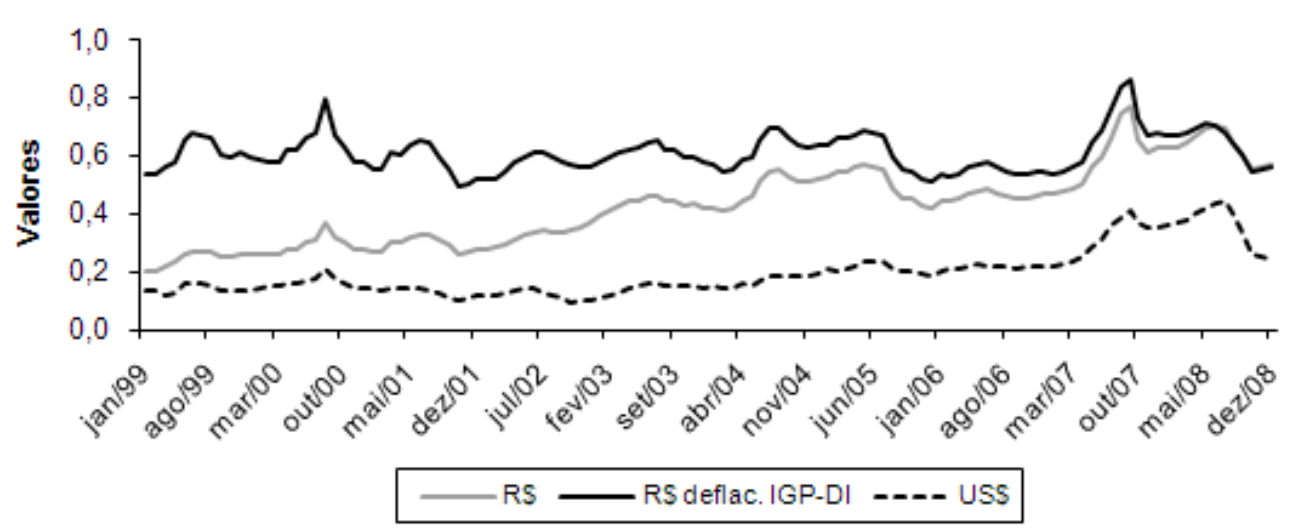

Figura 1. Série de preços do leite recebidos pelos produtores, estado do Paraná e Rio Grande do Sul, Janeiro de 1999 à Dezembro 2008. 
O preço do leite no estado do Paraná possui semelhanças com a do Rio Grande do Sul, tendo nível máximo no mês de agosto de 2007 com valor de $\mathrm{R} \$ 0,76$. Observa-se que para ambos os estados o índice geral de preço (IGP-DI) tem comportamento semelhante ao valor do produto, este valor refere-se à taxa de inflação de cada período, tendo influência no valor do leite ao produtor.

Os dados relativos à avaliação da série histórica do valor pago ao litro de leite e os respectivos coeficientes de variação nos estados do Rio Grande do Sul e Paraná são apresentados na Tabela1. Para a variável, valor pago ao litro de leite em reais, observou-se que o maior valor histórico no estado do Rio Grande do Sul foi para os anos de 2007 com média de R\$ 0,60 e 2008 com valor médio de $\mathrm{R} \$ 0,64$ e o menor valor foi observado para os anos de 1999 com valor de R $\$ 0,25$ e para os anos 2000 e 2001 com valor médio de R\$ 0,29. Apesar de não ser encontrada diferença significativa entre os anos de 2007 e 2008 ocorreu uma elevação do valor pago ao litro de leite de $6,67 \%$ causado entre outros fatores pela desvalorização do dólar. $\mathrm{O}$ valor do produto cotado na moeda americana era de U\$ 0,31 em 2007 e devido à desvalorização do cambio, foi significativamente $(\mathrm{P}<0,05)$ maior em $2008 \mathrm{com}$ valor médio de U\$ 0,36.

Tabela 1. Avaliação da série histórica do valor pago ao litro de leite nos estados do Rio Grande do Sul e Paraná.

\begin{tabular}{lccccccccccc}
\hline \multirow{2}{*}{ Valores } & \multicolumn{10}{c}{ Rio Grande do Sul } & \multirow{2}{*}{ CV\% } \\
\cline { 2 - 11 } & $\mathbf{1 9 9 9}$ & $\mathbf{2 0 0 0}$ & $\mathbf{2 0 0 1}$ & $\mathbf{2 0 0 2}$ & $\mathbf{2 0 0 3}$ & $\mathbf{2 0 0 4}$ & $\mathbf{2 0 0 5}$ & $\mathbf{2 0 0 6}$ & $\mathbf{2 0 0 7}$ & $\mathbf{2 0 0 8}$ & \\
\hline Reais & $0,25 \mathrm{a}$ & $0,29 \mathrm{ab}$ & $0,29 \mathrm{ab}$ & $0,33 \mathrm{~b}$ & $0,43 \mathrm{c}$ & $0,49 \mathrm{~cd}$ & $0,51 \mathrm{~d}$ & $0,46 \mathrm{~cd}$ & $0,60 \mathrm{e}$ & $0,64 \mathrm{e}$ & 10,03 \\
IGP-DI & $0,59 \mathrm{abc}$ & $0,62 \mathrm{bcd}$ & $0,57 \mathrm{ab}$ & $0,56 \mathrm{ab}$ & $0,60 \mathrm{abc}$ & $0,61 \mathrm{bcd}$ & $0,60 \mathrm{bcd}$ & $0,54 \mathrm{a}$ & $0,66 \mathrm{~d}$ & $0,64 \mathrm{~cd}$ & 7,86 \\
Dólar & $0,14 \mathrm{ab}$ & $0,16 \mathrm{ab}$ & $0,13 \mathrm{ab}$ & $0,12 \mathrm{a}$ & $0,14 \mathrm{ab}$ & $0,17 \mathrm{bc}$ & $0,20 \mathrm{~cd}$ & $0,21 \mathrm{~d}$ & $0,31 \mathrm{e}$ & $0,36 \mathrm{f}$ & 16,88 \\
\hline & \multicolumn{10}{c}{} & \multicolumn{10}{c}{ Paraná } & & & & & \\
Reais & $0,25 \mathrm{a}$ & $0,30 \mathrm{ab}$ & $0,28 \mathrm{ab}$ & $0,33 \mathrm{~b}$ & $0,44 \mathrm{c}$ & $0,48 \mathrm{c}$ & $0,49 \mathrm{c}$ & $0,46 \mathrm{c}$ & $0,61 \mathrm{~d}$ & $0,64 \mathrm{~d}$ & 11,06 \\
IGP-DI & $0,60 \mathrm{abc}$ & $0,64 \mathrm{bc}$ & $0,54 \mathrm{a}$ & $0,56 \mathrm{a}$ & $0,60 \mathrm{abc}$ & $0,60 \mathrm{abc}$ & $0,58 \mathrm{ab}$ & $0,54 \mathrm{a}$ & $0,67 \mathrm{c}$ & $0,64 \mathrm{bc}$ & 9,05 \\
Dólar & $0,14 \mathrm{ab}$ & $0,16 \mathrm{bc}$ & $0,12 \mathrm{ab}$ & $0,12 \mathrm{a}$ & $0,14 \mathrm{ab}$ & $0,17 \mathrm{bc}$ & $0,20 \mathrm{~cd}$ & $0,21 \mathrm{~d}$ & $0,32 \mathrm{e}$ & $0,36 \mathrm{e}$ & 18,13 \\
\hline
\end{tabular}

Médias seguidas de letras diferentes na mesma linha são diferentes $(\mathrm{P}<0,05)$ pelo teste de Tukey.

Desta forma os produtores gaúchos tiveram um acréscimo de $16,13 \%$ no valor do litro de leite em dólar, do ano de 2007 para 2008. O índice geral de preços - Disponibilidade interna (IGP-DI) é divulgado mensalmente pela Fundação Getúlio Vargas. Esse índice mede a evolução geral de preços na economia, criando assim uma medida da inflação nacional, apresentou valores significativamente maiores em 2004, 2005, 2007 e 2008 apresentando média geral de 0,63 com coeficiente de variação de $7,86 \%$. O menor valor significativo do IGP-DI foi no ano de 2006 com valor de 0,54 , desta forma, observado aumento de $15,63 \%$ entre 2006 e 2008.

O valor do litro de leite no estado do Paraná foi significativamente maior $(\mathrm{P}<0,05)$ em 2007 e 2008 com médias de $\mathrm{R} \$ 0,61$ e $\mathrm{R} \$ 0,64$ quando comparado com os demais anos avaliados. O menor valor foi encontrado para 1999 com valor médio de R \$ 0,25, o coeficiente de variação para esta variável foi de $11,06 \%$. O IGP-DI teve taxas significativamente semelhantes nos anos de 1999, 2000, 2003, 2004, 2007 e 2008. Sendo registrada uma redução no índice de $4,48 \%$ entre 2007 e 2008 . O valor do produto em dólar foi significativamente maior a partir de 2007, este fato pode ser devido à desvalorização do dólar em relação à moeda nacional. $\mathrm{O}$ valor médio combinado do leite de 1999 até 2006 foi de U\$ 0,16 e a média combinada dos anos de 2007 e 2008 foi de U\$ 0,34 com variação de 52,94\% no valor do leite. Segundo Andretta (2007), a pecuária no ano de 2005 teve incremento de $3 \%$ em relação ao ano de 2004 
e de $36,7 \%$ em relação ao ano de 1997 . Este grupo tem a maior participação no valor bruto em 2005 (40,73\%), superando, inclusive, o grupo de grãos.

As médias mensais históricas do valor pago ao litro de leite nos estados do Rio Grande do Sul e Paraná são apresentadas na Tabela 2. As maiores remunerações históricas no estado do Rio Grande do Sul foram significativamente $(\mathrm{P}<0,05)$ maiores nos meses de junho com valor de R $\$ 0,46$ e julho com valor médio de $\mathrm{R} \$ 0,47$. No estado do Paraná as maiores remunerações foram observadas em julho e agosto com valores de R\$ 0,47. Desta forma, foi evidenciada a sazonalidade da remuneração existente na cadeia leiteira, onde os maiores valores pagos ocorrem no período de inverno devido à menor oferta do produto, elevando a remuneração.

Tabela 2. Médias mensais históricas do valor pago por litro de leite nos estados do Rio Grande do Sul e Paraná, no período de janeiro 1999 à dezembro de 2008.

\begin{tabular}{ccccccc}
\hline \multirow{2}{*}{ Mês } & \multicolumn{3}{c}{ Rio Grande do Sul } & \multicolumn{3}{c}{ Paraná } \\
\cline { 2 - 6 } & Reais & IGP-DI & US \$ & Reais & IGP-DI & US \$ \\
\hline Janeiro & $0,39 \mathrm{a}$ & $0,56 \mathrm{a}$ & 0,18 & $0,37 \mathrm{a}$ & $0,53 \mathrm{a}$ & 0,17 \\
Fevereiro & $0,39 \mathrm{a}$ & $0,56 \mathrm{a}$ & 0,18 & $0,38 \mathrm{ab}$ & $0,54 \mathrm{a}$ & 0,17 \\
Março & $0,41 \mathrm{ab}$ & $0,58 \mathrm{abc}$ & 0,18 & $0,40 \mathrm{abc}$ & $0,57 \mathrm{abc}$ & 0,18 \\
Abril & $0,42 \mathrm{ab}$ & $0,60 \mathrm{abc}$ & 0,19 & $0,42 \mathrm{abcd}$ & $0,60 \mathrm{abcd}$ & 0,19 \\
Maio & $0,44 \mathrm{ab}$ & $0,62 \mathrm{abc}$ & 0,20 & $0,45 \mathrm{bcd}$ & $0,63 \mathrm{bcd}$ & 0,21 \\
Junho & $0,46 \mathrm{~b}$ & $0,65 \mathrm{bc}$ & 0,21 & $0,46 \mathrm{~cd}$ & $0,65 \mathrm{~cd}$ & 0,21 \\
Julho & $0,47 \mathrm{~b}$ & $0,66 \mathrm{c}$ & 0,22 & $0,47 \mathrm{~d}$ & $0,66 \mathrm{~d}$ & 0,22 \\
Agosto & $0,46 \mathrm{~b}$ & $0,65 \mathrm{bc}$ & 0,21 & $0,47 \mathrm{~d}$ & $0,65 \mathrm{~d}$ & 0,22 \\
Setembro & $0,45 \mathrm{ab}$ & $0,61 \mathrm{abc}$ & 0,20 & $0,45 \mathrm{~cd}$ & $0,63 \mathrm{bcd}$ & 0,21 \\
Outubro & $0,42 \mathrm{ab}$ & $0,58 \mathrm{ab}$ & 0,18 & $0,43 \mathrm{abcd}$ & $0,59 \mathrm{abc}$ & 0,19 \\
Novembro & $0,42 \mathrm{ab}$ & $0,57 \mathrm{a}$ & 0,18 & $0,42 \mathrm{abcd}$ & $0,57 \mathrm{abc}$ & 0,18 \\
Dezembro & $0,42 \mathrm{ab}$ & $0,57 \mathrm{a}$ & 0,18 & $0,42 \mathrm{abcd}$ & $0,56 \mathrm{ab}$ & 0,18 \\
\hline Média Geral & 0,43 & 0,60 & 0,19 & 0,43 & 0,60 & 0,19 \\
\hline
\end{tabular}

Médias seguidas de letras diferentes na mesma coluna são diferentes $(\mathrm{P}<0,05)$ pelo teste de Tukey.

O índice geral de preços que atua como um marcador da inflação nacional apresentou diferenças significativas $(\mathrm{P}<0,05)$ para os estados do Rio Grande do Sul com valor médio geral de 0,60 . No estado do Paraná foram encontradas diferenças entre os meses e o valor do índice, apresentando valores significativamente maiores para os meses de abril à setembro com valor médio de 0,64. Esta variação mensal no valor do IGP-DI pode ter influenciado na variação do valor do produto em reais. $\mathrm{O}$ produto cotado em dólar não apresentou diferenças significativas $(\mathrm{P}<0,05)$ para as médias mensais nos estados. No Rio Grande do Sul o leite teve um valor médio geral de U\$ 0,19 com coeficiente de variação de $16,88 \%$ e no Paraná o valor médio foi de U\$ 0,19 apresentando coeficiente de variação de $18,13 \%$.

A análise de trilha, mostrando o desdobramento das correlações em componentes de efeito diretos e indiretos envolvendo a variável dependente principal valor pago ao litro do leite (Rleite) e as variáveis independentes explicativas índice geral de preços, taxa de câmbio e valor da saca da soja, milho e trigo são apresentadas na Tabela 3. 
Tabela 3. Estimativa dos efeitos diretos e indiretos do valor pago ao litro de leite nos estados do Paraná e Rio Grande do Sul e seus componentes primários (IGP-DI, Câmbio, Rsoja, Rmilho e Rtrigo).

\begin{tabular}{|c|c|c|c|}
\hline Variável & Via & Paraná & Rio Grande do Sul \\
\hline Efeito direto de IGP-DI & & 0,3257 & 0,3257 \\
\hline Efeito indireto de IGP-DI & Via Câmbio & $-0,074$ & $-0,0070$ \\
\hline Efeito indireto de IGP-DI & Via Rsoja & $-0,0288$ & $-0,0275$ \\
\hline Efeito indireto de IGP-DI & Via Rmilho & 0,0969 & 0,0773 \\
\hline Efeito indireto de IGP-DI & Via Rtrigo & 0,1564 & 0,1786 \\
\hline Total - Diretos e Indiretos & & 0,5428 & 0,5471 \\
\hline Efeito direto de Câmbio & & 0,0365 & 0,0350 \\
\hline Efeito indireto de Câmbio & Via IGP-DI & $-0,0660$ & $-0,0649$ \\
\hline Efeito indireto de Câmbio & Via Rsoja & $-0,0889$ & $-0,0854$ \\
\hline Efeito indireto de Câmbio & Via Rmilho & $-0,1087$ & $-0,1036$ \\
\hline Efeito indireto de Câmbio & Via Rtrigo & 0,1896 & 0,1882 \\
\hline Total - Diretos e Indiretos & & $-0,0376$ & $-0,0307$ \\
\hline Efeito direto de Rsoja & & $-0,1835$ & $-0,1763$ \\
\hline Efeito indireto de Rsoja & Via IGP-DI & 0,0512 & 0,0508 \\
\hline Efeito indireto de Rsoja & Via Câmbio & 0,0177 & 0,0170 \\
\hline Efeito indireto de Rsoja & Via Rmilho & 0,1373 & 0,1308 \\
\hline Efeito indireto de Rsoja & Via Rtrigo & 0,6541 & 0,6494 \\
\hline Total - Diretos e Indiretos & & 0,6767 & 0,6716 \\
\hline Efeito direto de Rmilho & & 0,1807 & 0,1722 \\
\hline Efeito indireto de Rmilho & Via IGP-DI & 0,1746 & 0,1462 \\
\hline Efeito indireto de Rmilho & Via Câmbio & $-0,0219$ & $-0,0211$ \\
\hline Efeito indireto de Rmilho & Via Rsoja & $-0,1394$ & $-0,1340$ \\
\hline Efeito indireto de Rmilho & Via Rtrigo & 0,5191 & 0,5154 \\
\hline Total - Diretos e Indiretos & & 0,7131 & 0,6787 \\
\hline Efeito direto de Rtrigo & & 0,7698 & 0,7643 \\
\hline Efeito indireto de Rtrigo & Via IGP-DI & 0,0662 & 0,0761 \\
\hline Efeito indireto de Rtrigo & Via Câmbio & 0,0090 & 0,0863 \\
\hline Efeito indireto de Rtrigo & Via Rsoja & $-0,1559$ & $-0,1498$ \\
\hline Efeito indireto de Rtrigo & Via Rmilho & 0,1218 & 0,1161 \\
\hline Total - Diretos e Indiretos & & 0,8109 & 0,8153 \\
\hline Coeficiente de Determinação & & 0,8044 & 0,7987 \\
\hline
\end{tabular}


O efeito direto do índice geral de preço (IGP-DI) no valor pago ao litro do leite no estado do Paraná foi de 0,3257 e observou-se o maior efeito indireto foi para o Rtrigo com valor de 0,1564. Este fato pode ser atribuído à alta ocorrida no valor do trigo devido às crescentes exportações ocasionando a redução no volume do produto no mercado. $\mathrm{O}$ total de efeitos diretos e indiretos foi de 0,5428. Para o estado do Rio Grande do Sul foi observado relações semelhantes, o total de efeito diretos e indiretos foi de 0,5471 , sendo o efeito direto do IGP-DI de 0,3257 e o principal efeito indireto foi em relação ao Rtrigo com correlação de 0,1786 . O índice geral de preços apresenta maior correlação direta com a variável Rleite em ambos os estados.

A taxa de câmbio apresentou baixa correlação direta com o valor pago ao leite, porém apresenta influências indiretas nas demais variáveis independentes. No estado do Paraná o efeito direto do câmbio foi de apenas 0,0365 , mas teve efeito indireto principalmente na variável Rtrigo com valor de 0,1896 e registrando um total de efeitos diretos e indiretos de $-0,0376$. No Rio Grande do Sul o total de efeito direto e indireto foi de apenas $-0,0307$ com maior correlação positiva indireta na variável Rtrigo com valor de 0,1882. Mas a taxa de câmbio apresentou efeitos indiretos negativos de $-0,0854$ para Rsoja e -0,1036 para Rmilho, ocasionando o baixo valor de correlação total da variável, indicando que o valor da saca de soja (Rsoja) apresentou um efeito direto negativo no valor do leite.

No Paraná o total de efeitos direto e indiretos da Rsoja foi de 0,6767 , este fato é coerente, pois a soja é um dos principais componentes de suplementos concentrados para a produção leiteira e tem participação efetiva no valor pago aos produtores pelo leite. $\mathrm{O}$ efeito direto no estado paranaense foi de $-0,1835$ e o principal correlação indireta foi com a variável Rtrigo com valor de 0,6541. Assim mesmo observando-se correlação direta negativa a variável Rsoja apresenta grandes correlações positivas com as demais variáveis ocasionando uma correlação total positiva. O Rio Grande do Sul teve relações semelhantes com o estado do Paraná, com o efeito direto negativo de $-0,1763$ da Rsoja para o Rleite. Observou-se a maior correlação indireta para a variável Rtrigo com valor de 0,6494 , desta forma ocasionando em um efeito total de 0,6716.

No Paraná o total de efeitos diretos e indiretos da variável Rmilho no valor do leite é de 0,7131. Indicando que o valor do milho tem influencia direta no valor pago pelo litro de leite ao produtor, pois juntamente com a soja formam a base da suplementação concentrada utilizada na produção leiteira. $\mathrm{O}$ efeito direto observado do Rmilho foi de 0,1807 e o principal efeito indireto 0,5191 ocorreu com a variável Rtrigo. No Rio Grande do Sul o total de efeitos direto e indiretos do Rmilho foi de 0,6787, onde o maior efeito indireto 0,5154 para a variável Rtrigo. Em ambos os estados os principais efeitos na variável Rleite são de característica indireta.

O valor da saca de trigo (Rtrigo) no estado do Paraná teve um elevado efeito direto de 0,7698 no valor do leite. Sendo o principal componente do total de efeitos diretos e indiretos 0,8109 na Rleite. No Rio Grande do Sul o efeito direto da variável Rtrigo foi 0,7643 sendo o principal componente do total dos efeitos direto e indiretos com valor de 0,8153. Na última safra, 2007/08, o Brasil produziu cerca de 3.832 toneladas de trigo, um crescimento de $71,50 \%$ em relação à safra 2006/07, o que se deve principalmente à elevação da produtividade de $65,70 \%$, enquanto a área para cultivo cresceu apenas 3,5\%, segundo dados da Companhia Nacional de Abastecimento (2008).

O coeficiente de determinação do modelo da análise de trilha $\left(\mathrm{R}^{2}\right)$ apresentou-se elevado nos estados do Paraná e Rio Grande do Sul, com valores de 0,8044 e 0,7987 , respectivamente. Indicando que as variáveis independentes explicativas IGP-DI, Cambio, Rsoja, Rmilho e Rtrigo, explicaram grande parte da variação da variável básica Rleite. 


\section{Conclusões}

Observou-se variação da remuneração ao litro de leite produzido nos estados do Paraná e Rio Grande do Sul, e que os efeitos diretos e indiretos das variáveis analisadas tiveram correlação com a variável Rleite.

\section{Referências}

ANDRETTA, G. M. A. C. Valor bruto da produção agropecuária paranaense de 2005. Curitiba: SEAB/ DERAL/DEB, 2007.

COMPANHIA NACIONAL DE ABASTECIMENTO - CONAB. Central de informações agropecuárias. Disponível em: $<\mathrm{http}: / /$ www.conab.gov.br $>$. Acesso em: 1 fev. 2008.

CRUZ, C. D.; REGAZZI, A. J. Modelos biométricos aplicados ao melhoramento genético. Viçosa : UFV, 1997. $390 \mathrm{p}$.

DUNTEMAN, G. H. Introduction to multivariate analysis. Beverly Hills : Sage Publications, 1984.
FALCONER, D. S. Introdução à genética quantitativa. Viçosa: UFV, 1987.

GOMES, S. T. Diagnóstico e perspectivas da produção de leite no Brasil. In: VILELA, D.; BRESSAN, M.; CUNHA, A. S. Cadeia de lácteos no Brasil: restrições ao seu desenvolvimento. Brasília: MCT/CNPq, Juiz de Fora: EMBRAPA Gado de Leite, 2001. p. 21-37.

UNIVERSIDADE FEDERAL DE VIÇOSA. Sistemas de Análises Estatísticas e Genéticas - SAEG: versão 9.1. Viçosa: UFV, 2007. Manual do Usuário.

VENCOVSKY, R.; BARRIGA, P. Genética biométrica no fitomelhoramento. Ribeirão Preto: Revista Brasileira de Genética, 1992.

VILELA, D. A importância econômica, social e nutricional do leite. Revista Batavo, Castro, n. 111, dez. 2001/jan. 2002.

WRIGHT, S. Correlation and causation. Journal of Agricultural Research, Washington, v. 20, n. 7, p. 557585, 1921.

WRIGHT, S. Theory of path coefficients. Genetics, New York, v. 8, p. 239-285, 1923. 